Review

\title{
Surface Science Studies of Gas Sensing Materials: $\mathrm{SnO}_{2}$
}

\author{
Matthias Batzill \\ Department of Physics, University of South Florida, Tampa, FL 33620, USA \\ E-mail: mbatzill@cas.usf.edu
}

Received: 26 September 2006 / Accepted: 16 October 2006 / Published: 17 October 2006

\begin{abstract}
This review is an attempt to give an overview on how surface science studies can contribute to a fundamental understanding of metal oxide gas sensors. In here tin dioxide is used as a model system for metal oxide gas sensor materials and we review surface science studies of single crystal SnO2. The composition, structure, electronic and chemical properties of the (110) and (101) surfaces is described. The influence of compositional changes as a function of the oxygen chemical potential on the electronic surface structure and the chemical properties is emphasized on the example of the (101) surface. The surface chemical properties are discussed on the example of water adsorption. It is shown the chemical and gas sensing properties depend strongly on the surface composition.
\end{abstract}

Keywords: surface science, single crystal, metal oxide surfaces, photoemission, scanning tunneling microscopy.

\section{Introduction}

Metal oxides, such as $\mathrm{ZnO}, \mathrm{In}_{2} \mathrm{O}_{3}$, $\mathrm{SnO}_{2}$, etc., have been studied extensively for their gas response to oxidizing and reducing gases. Generally, gas sensitive metal oxide materials are porous thick films. In a gas sensor, molecular adsorption or reaction of molecules with pre-adsorbed chemical species, results in a change of the electrical conductivity. This conductivity change can be easily detected and is often used as the gas response signal. Thus, because the fundamental process is the adsorption and reaction of molecules at an oxide surface, solid state gas sensing is a surface science problem. The basic mechanism that causes the conductivity of the material to change upon adsorption or reaction of molecules at the surface is a consequence of the chemical state of the adsorbed species. Charge transfer from the oxide to the chemi- or ionosorbed molecule causes a net surface charge. As a consequence of this surface charge a space charge region is induced in the surface region of the oxide- 
semiconductor that balances the charges at the surface. [1,2] This space charge region is described by a band bending of the energy levels- or a change in the charge carrier chemical potential, i.e. a shift in the Fermi level- and thus causes an accumulation or depletion of charge carriers depending on the sign of the surface charge. Consequently, a change in charge carrier concentration due to adsorbate induced band bending is the cause for the gas response in conductivity sensitive solid state gas sensors. Incidentally, the three oxides mentioned above, are all n-type conductors with high electron mobilitythis property makes them also useful materials for transparent electrodes in optoelectronic devices and other applications.

Practical gas sensors are often porous materials. The microstructure of these materials causes additional effects associated with grain boundaries and necks between grains that may amplify the gas sensitivity. These effects are, however, secondary and are not fundamental to the gas sensing mechanism. Gas responses on nanoscopic single crystalline materials, [3] without the presence of grains, can be as large as or larger than those in the common porous thick film gas sensors as long the length scale of these single crystalline materials are of the order of the space charge region, which can extend several micrometers.

In the context of charged surface species, pre-adsorbed oxygen species play an outstanding role for the detection of combustible gases. From electron spin resonance (ESR) measurements it has been proposed that adsorbed oxygen can be present in various chemical species transferring electrons from gas sensing oxide to the chemisorbed oxygen according to the following process: [4]

$$
\mathrm{O}_{2} \text { (gas) } \Leftrightarrow \mathrm{O}_{2}(\mathrm{ad}) \Leftrightarrow \mathrm{O}_{2}^{-}(\mathrm{ad}) \Leftrightarrow \mathrm{O}^{-}(\mathrm{ad}) \Leftrightarrow \mathrm{O}^{2-} \text { (ad) } \Leftrightarrow \mathrm{O}^{2-} \text { (lattice) }
$$

The temperature dependence of the different species was examined by Chang. [5] He observed a transition temperature at $150{ }^{\circ} \mathrm{C}$. Below which oxygen is mainly present as $\mathrm{O}_{2}{ }^{-}$and above chemisorbed oxygen in the from of $\mathrm{O}^{-}$or $\mathrm{O}^{2-}$ is present. This change in chemistry was correlated to a decrease in sample conductivity that occurred at around $160{ }^{\circ} \mathrm{C}$. From these observations the following basic mechanism for detection of combustible gases seems plausible: If the gas sensor is operated under ambient conditions it can be assumed that chemically adsorbed oxygen species are present at the surface. Combustible gases may react with these oxygen species and thus result in depletion of charged surface oxygen which in turn increases the conductivity of the gas sensing material. In this way combustible gases may not directly interact with the gas sensing material but its presence controls the concentration of pre-adsorbed oxygen, which controls the surface charge and thus the conductivity of the gas sensor.

In the traditional surface science approach single crystal surfaces are studied under ultra high vacuum conditions. Such a stringent environment is necessary for investigating fundamental processes because only in a vacuum in the $10^{-10}$ mbar regime it can be assured that the surface stays reasonably clean for an extended period of time. This then allows the investigation of the clean surface and a subsequent exposure to pure gases to investigate their impact on the surface property. A well defined system is essential for understanding fundamental processes at gas sensing surfaces, however, there are limitations and pitfalls in the surface science approach that one needs to keep in mind if one wants to 
project results of surface science studies to realistic applications of these materials in gas sensing devices.

This review is intended to give an overview of basic surface science studies of gas sensing metal oxides on the example of $\mathrm{SnO}_{2}$. Here we define surface science as studies on single crystal surfaces under well defined atmospheres, mainly ultra high vacuum. It is assumed that most readers are not surface scientists and thus an attempt is being made to convey the benefits of a rigorous surface science approach to a broader gas sensing community. In the next section some general remarks are being made on how surface science studies can contribute to a fundamental understanding of gas sensing materials and the challenges surface scientists face in comparing model system results to 'real world' applications. After this prelude two concrete examples of surface science studies are being discussed. The first is the structural and compositional properties of $\mathrm{SnO}_{2}(101)$ and (110) surfaces and the second study is the adsorption of water onto these surfaces. We will conclude with an outlook into future surface science studies.

\section{Limits and Challenges of Surface Science for Understanding Gas Sensing Materials}

To understand fundamental processes a system must be well defined at the length scale these processes take place. i.e. the molecular level in the case of solid state gas sensing. To have a well defined system two conditions have to be fulfilled. Firstly the system can be prepared so that it is void of any molecular contamination and secondly one must have the techniques necessary to characterize the system at the appropriate length scale and concentration-level. Ultra high vacuum based surface science can deliver these prerequisites and this is largely the appeal of this approach. The vacuum conditions allow to prepare surfaces with high cleanliness and the low background pressure in an ultrahigh vacuum chamber ascertains the cleanliness of the surface long enough to conduct experiments.

In addition to the guarantee of pristine conditions, vacuum also allows the use of atoms, ions, electrons, and photons for a thorough characterization of the surface structure, electronic, and chemical properties.

Single crystal surfaces are obviously better defined than the materials used in gas sensing applications. However, even a single crystal surface can have complex defect structures, e.g. steps, atomic vacancies, etc. Often these defects play an important role in the surface chemistry of oxides. Atomic scale characterization by for instance scanning tunneling microscopy allows in principle the investigation of these defects and also the interaction of these defects with gases. Although there is some work done on the surface defect structures of $\mathrm{TiO}_{2}$ - a model oxide in the surface science community [6]- there is only limited work so far on surface sensitive gas sensing oxides. The surface science approach is the only reliable way to obtain information of molecular scale interaction with solids.

Despite the overwhelming advantages UHV surface science investigations affords there are also 'risks' associated by studying a system at a condition that is very different from the environment encountered in the applications one hopes to understand. It is apparent that the difference in pressure between studies in vacuum to that under ambient pressure conditions alters the thermodynamic conditions. To illustrate these differences we consider the simple Lenard-Jones model for adsorption. 
In this model the rate of chemisorption is given by an activation barrier between a physiosorbed state and the chemisorbed state and an activation barrier of desorption as is illustrated in Figure 1(a). The rate of chemisorption $\mathrm{d} \Theta / \mathrm{dt}$ is expressed as the difference in adsorption and desorption rates:

$$
\mathrm{d} \Theta / \mathrm{dt}=\mathrm{k}_{\mathrm{ads}} \exp \left\{-\Delta \mathrm{E}_{\mathrm{A}} / \mathrm{kT}\right\}-\mathrm{k}_{\mathrm{des}} \Theta \exp \left\{-\left(\Delta \mathrm{E}_{\mathrm{A}}+\Delta \mathrm{H}_{\mathrm{chem}}\right) / \mathrm{kT}\right\}
$$

where $\Delta \mathrm{E}_{\mathrm{A}}$ is the activation barrier for chemisorption and $\Delta \mathrm{H}_{\text {chem }}$ is the heat of chemisorption. Under steady state conditions, i.e. $\mathrm{d} \Theta / \mathrm{dt}=0$, the coverage is given by:

$$
\Theta=\mathrm{k}_{\mathrm{ads}} / \mathrm{k}_{\mathrm{des}} \exp \left\{\Delta \mathrm{H}_{\mathrm{chem}} / \mathrm{kT}\right\}
$$

Thus generally the coverage will decrease with temperature. At low temperatures the molecules are, however, trapped in a physisorbed state and cannot overcome the activation barrier $\Delta \mathrm{E}_{\mathrm{A}}$. This results in a maximum coverage of chemisorbed moieties at a temperature $T_{\max }$ illustrated in Fig 1(b).

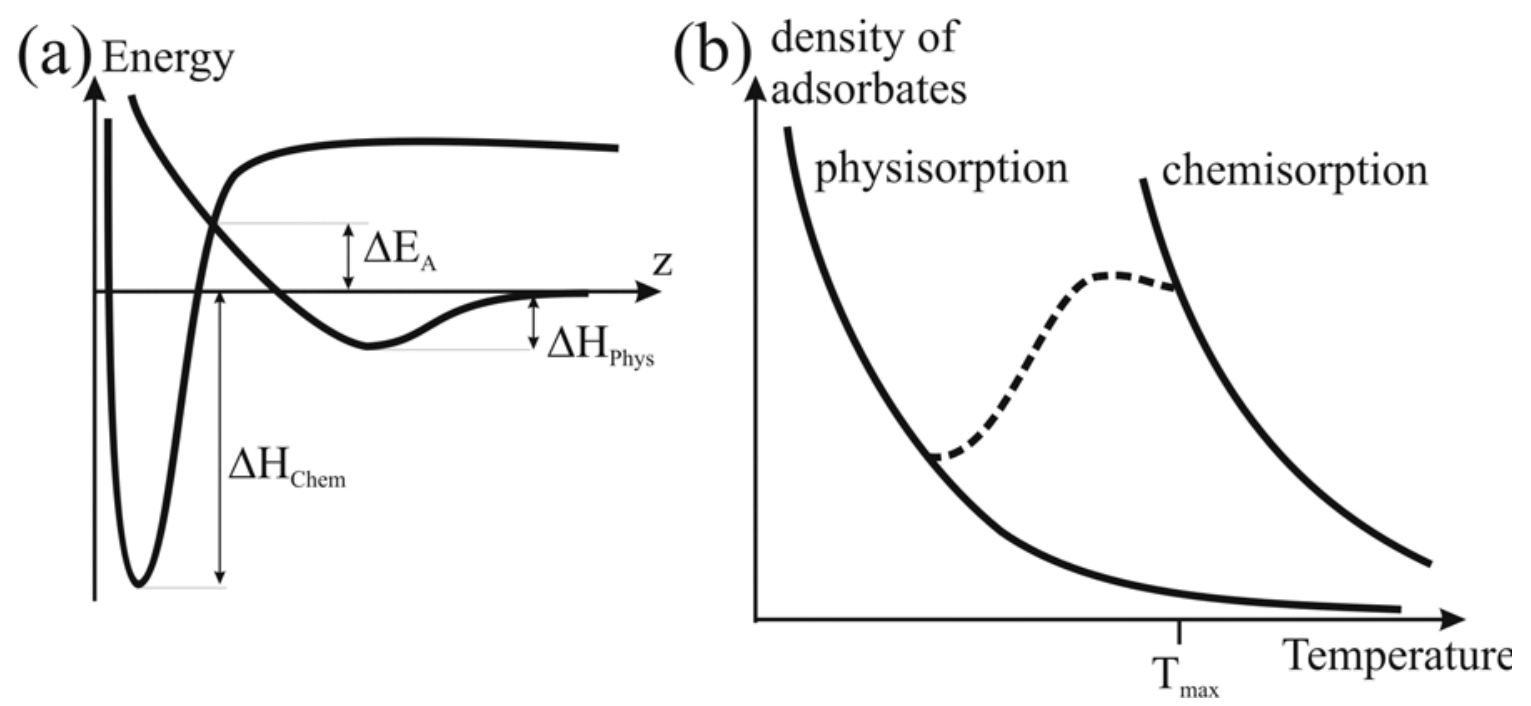

Figure 1. (a) Lenard-Jones model for physisorption and chemisorption of molecules. Typical adsorption isobars are shown in (b). The solid lines are equilibrium physisorption and chemisorption isobars, the dashed line represents irreversible chemisorption. A maximum coverage of chemisorbed molecules is obtained at $T_{\max }$. (reproduced with permission from ref. [37]).

The Lenard-Jones model assumes an equilibrium between adsorbed molecules and molecules in the gas phase. In UHV experiments this is rarely the case, though. Instead molecules are adsorbed at low temperatures where their thermal activation is too low to overcome the desorption barrier and thus molecules are kinetically trapped at the surface. Adsorption at low temperatures thus allows simulating high coverage conditions with adsorbates that could occur in equilibrium with the gas phase at high pressures and at elevated temperatures. However, kinetic barriers for reactions at surfaces (e.g. chemisorption of physisorbed species, or reaction between adsorbates), make it apparent that 
adsorption at low temperatures and pressures is not equivalent to conditions encountered in high pressure conditions at elevated temperatures. The differences in UHV studies to "real world" applications are often dubbed the "pressure gap" in heterogeneous catalysis community. Thus in solid state gas sensors, for which, as we discussed above, activation of e.g. oxygen to different charge states plays an important role, studies at low pressures may not allow to capture all the complexity that occurs at oxide surfaces at elevated pressure and temperature. Consequently, it will be necessary to ultimately combine UHV studies with high pressure studies to obtain a full picture. Only limited surface science characterization can be performed at high pressures, but methods like infrared spectroscopy, mass-spectroscopy of the gas content (reaction products), STM, or grazing incidence Xray diffraction allows the characterization of chemical reactions and surface structure under these conditions. Advances in the design of differentially pumped electron analyzers are now also allowing photoelectron spectroscopy with the sample at elevated pressures. Although there has been a lot of technical advancements in the characterization of surfaces under "environmental" conditions in recent years, there have yet surface studies to be performed on single crystal gas sensing oxides in-situ at elevated pressure.

In the following we focus on the current understanding of the surface science of $\mathrm{SnO}_{2}$ single crystals. $\mathrm{SnO}_{2}$ has been one of the first metal oxide materials that have been considered as a gas sensing material and is still considered the prototypical solid state gas sensor. In this review we focus on studies of clean surfaces under UHV conditions and on water adsorption on these surfaces under UHV conditions. Water is omnipresent in any ambient applications and is known to cause a strong conductivity increase of the gas sensing material. The interaction of water with solid surfaces is a long standing problem in surface science. This and its known gas response make it an ideal probe molecule for fundamental surface science investigations.

\section{Clean low index surfaces of $\mathrm{SnO}_{2}$}

Single crystals expose mainly $\{110\}$ and $\{101\}$ faces. This is apparent from the photograph shown in figure 2. Consequently these two faces are the most studied single crystal surfaces and we address their properties in the following.

\subsection{The (110) surface}

The (110) surface has been studied by many investigators both theoretically $[7,8,9,10,11,12,13]$ as well as experimentally.[14,15,16,17,18,19,20,21,22,23,24,25] Complex surface reconstructions are being observed on this surface. The structure depends critically on the sample preparation and an easy reduction of $\mathrm{SnO}_{2}$ appears to result often in a sub-stoichiometric oxygen concentration at the surface. A characterization of the surface structure and composition is the prerequisite for any further surface science investigation. Therefore we describe what is known about the surface properties of the (110) surface. The complexity of the (110) surface challenges the preparation of well defined surfaces, on the other hand it may provide opportunities for the understanding of chemical processes by preparing surfaces with different compositions and anion-cation coordination. The chemistry of $\mathrm{SnO}_{2}(110)$ surfaces with different composition was extensively investigated by Cox and co-workers.[26,27,28] In the following we discuss the different surface structures that are observed on the (110) surface. 
Surface preparation under UHV conditions (for samples that are not easily cleaved) is generally performed by ion-sputtering to remove contaminations and subsequent high temperature annealing to heal sputter damage and reformation of a crystalline surface. Ion sputtering preferentially removes lighter surface constituents and thus an oxygen depleted surface is formed after sputtering. This is common for many oxides but subsequent annealing generally reforms the bulk composition at the surface. The situation on the $\mathrm{SnO}_{2}(110)$ surface appears, however, to be more complicated than for most other oxides. Annealing of the ion sputtered surface results in a series of complex surface reconstructions of oxygen deficient structures.

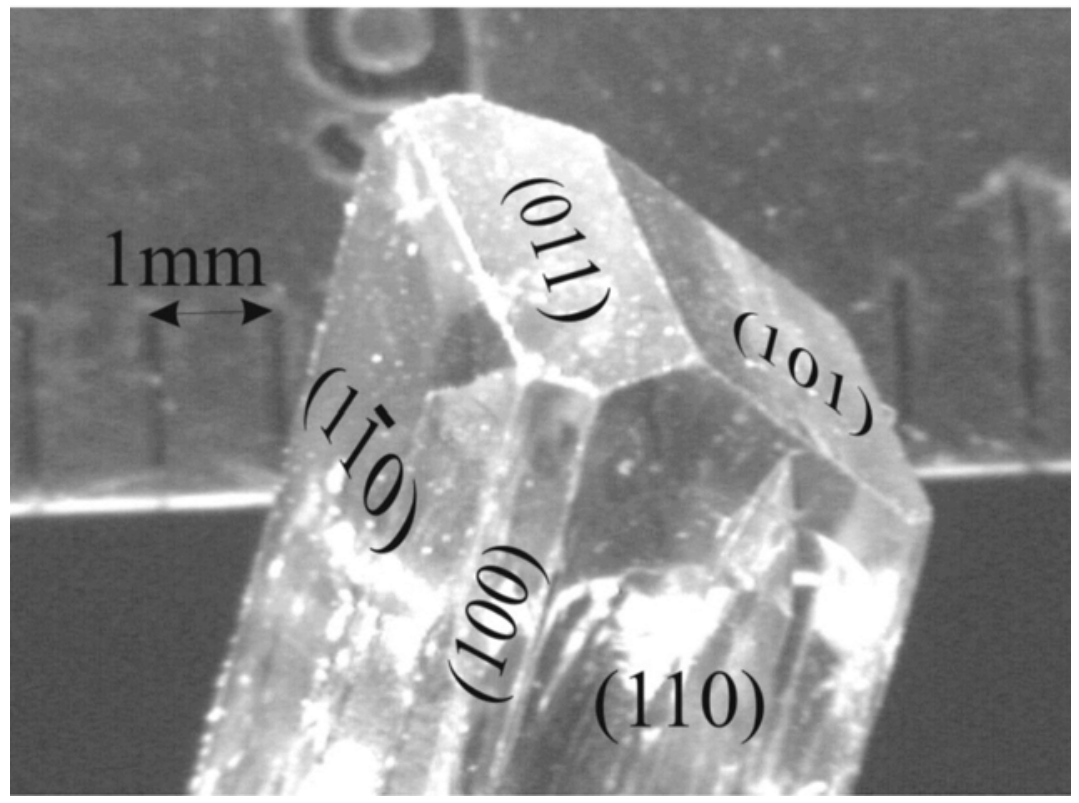

Figure 2. Photograph of a $\mathrm{SnO}_{2}$ single crystal grown by vapor phase transport. (reproduced with permission from ref. [36]).

The sequence of super-structures that are observed with increasing annealing temperatures has been reported by various investigators. First, a diffuse $\mathrm{c}(2 \times 2)$ structure is observed in low energy electron diffraction (LEED), followed by a mixed $c(2 \times 2)$ and $4 \times 1$ structure, a pure $4 \times 1$ structure, a " $1 \times 1$ " structure, and finally by a $1 \times 2$ superstructure. Figure 3 shows scanning probe images of the surface morphologies of some of these structures. From these studies it is apparent that the $4 \times 1$ structure exhibits the best organized surfaces with large flat terraces. The " $1 \times 1$ "structure turns out to be a disordered surface with the $1 \times 1$ LEED spots most likely originating from the subsurface region. [29] The $1 \times 2$ structure has large flat terraces; however, the surface is quite defective. In another study, using photoelectron spectroscopy, in the Cooper minimum for Sn, Themlin et al. [30] demonstrated that the $\mathrm{Sn} / \mathrm{O}$ ratio is high after $\mathrm{Ar}^{+}$-sputtering and that even after annealing to $1000 \mathrm{~K}$ the bulk stoichiometry is not recovered at the surface. Therefore all the above described surface reconstructions are strongly oxygen deficient. This is interesting because it illustrates that new periodic surface structures can be formed on the (110) surface by depleting the surface of oxygen. It is not clear, however, if similar structures form if a stoichiometric surface is exposed to strongly reducing 
atmosphere (e.g. hydrogen). Consequently, the relevance of some of these ordered surface structures for gas sensing applications is not conclusively established.
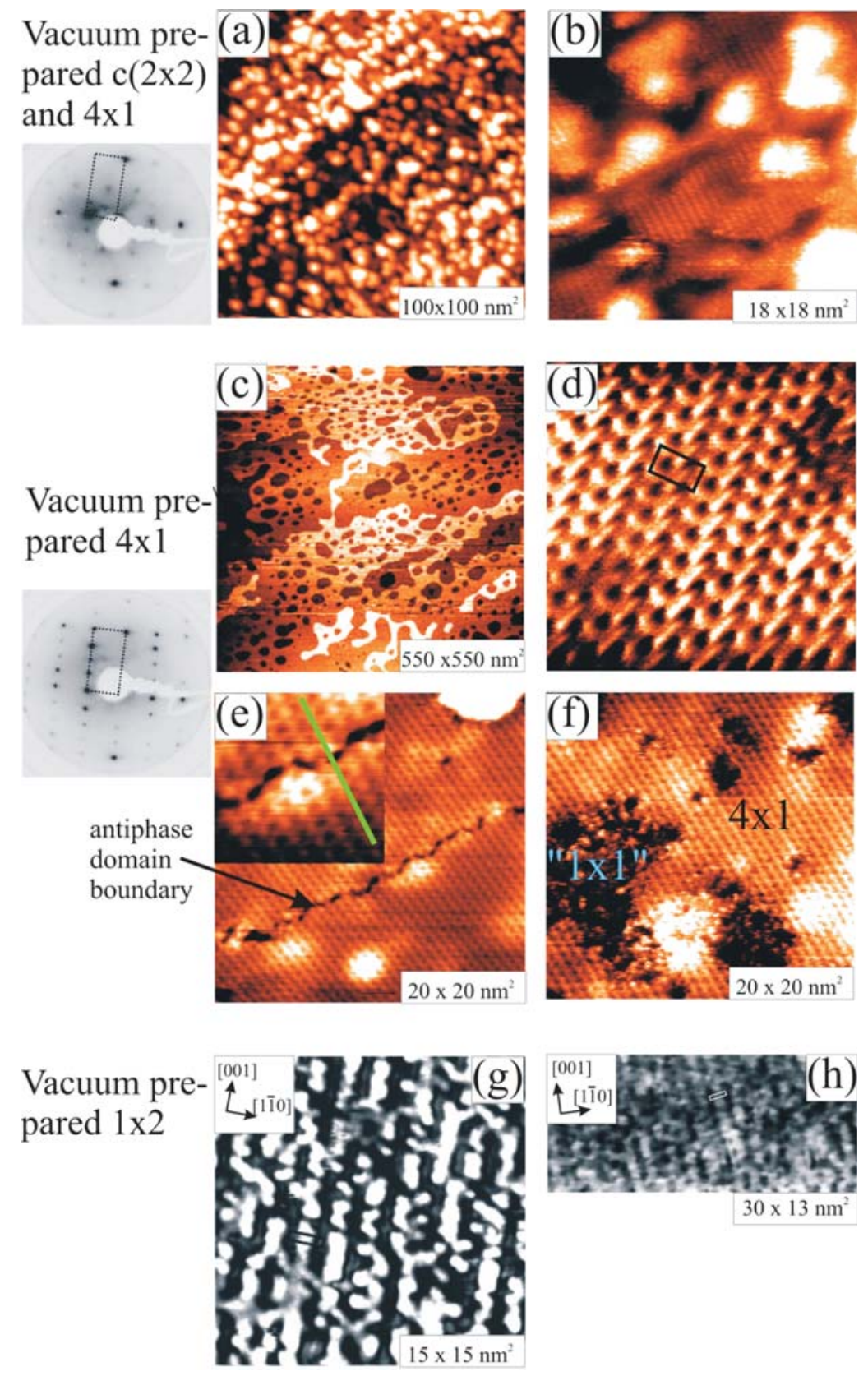

Figure 3. Overview of surface structures obtained for the $\mathrm{SnO}_{2}(110)$ surface for different annealing temperatures in UHV after ion sputtering. LEED patterns and STM images are shown. The 1x1 unit cell is indicated in the LEED patterns. (a) and (b) show STM images for low annealing temperatures surfaces are characterized by a coexistence of $4 \times 1$ and $c(2 \times 2)$ domains. The surface exhibits a high density of monolayer high islands that presumably are the $\mathrm{c}(2 \mathrm{x} 2)$ domains while the "substrate" exhibits mainly 4x1 domains. (c) -(f) show 4x1 reconstructed surfaces. In (f) domains of disordered structure appear. (g) and (h) show 1x2 reconstructed surfaces obtained after high temperature annealing (note that (g) is an AFM image). (reproduced with permission from ref. [29], ref. [25], and ref. [37]). 
In order to prepare a $1 \times 1$ surface with a high oxygen concentration ion sputtered $\mathrm{SnO}_{2}(110)$ surfaces have to be annealed in an oxidizing gas $\left(\mathrm{O}_{2}\right.$ [31] or $\mathrm{N}_{2} \mathrm{O}$ [32,33]) or exposed to an oxygen plasma [34] at elevated temperature. The surface structure that is prepared by exposing the surface to $10^{-3}$ mbar $\mathrm{O}_{2}$ at elevated temperature [29] is shown in figure 4. The surface exhibits a $1 \times 1$ LEED pattern and in STM it appears to be a stoichiometric bulk truncation with a high degree of defects indicating a low defect formation energy. This is likely a consequence of the easy reduction of $\mathrm{SnO}_{2}$ surfaces and possibly different competing meta-stable surface structures. In the next-subsection this easy reduction is discussed in more detail on the example of the (101) surface. The (110) surface is likely to behave in a similar fashion and easily loose surface oxygen, however, the different atomic coordination of the (101) surface allows for an adaptation of a reduced surface composition without restructuring of the surface atoms. The easy loss of surface oxygen can be understood by the dual valency of Sn that allows the transformation of surface tin from $\mathrm{Sn}$ (IV) to a $\mathrm{Sn}$ (II) valence state if oxygen is removed.
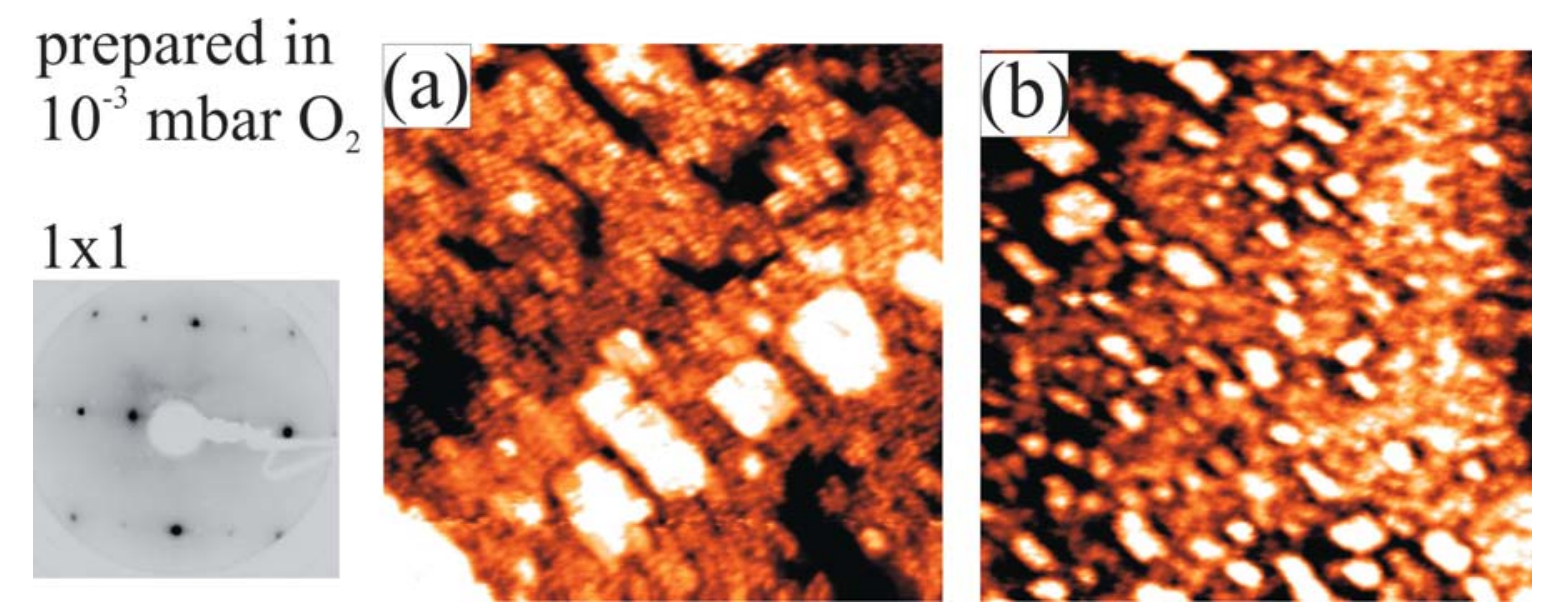

Figure 4. LEED pattern and STM images of $\mathrm{SnO}_{2}$ (110) surfaces prepared by (a) annealing in $10^{-3}$ mbar $\mathrm{O}_{2}$ and (b) after subsequent annealing to $800 \mathrm{~K}$ in vacuum. The scan area of the STM images is 100x $100 \mathrm{~nm}^{2}$. (reproduced with permission from ref. [29]).

Surface reduction by vacuum annealing has been studied on a (110) surface that had been oxidized in a high pressure cell at $10 \mathrm{mbar}_{2}$ at $\sim 500 \mathrm{~K}$. Annealing of such a fully oxidized sample in vacuum initially results in the loss of oxygen from the $1 \times 1$ structure without indication of the formation of a superstructure in qualitative LEED. The loss of oxygen has been observed by low energy He-ion scattering spectroscopy. [29,31] Annealing to $900 \mathrm{~K}$ results in the transformation to a $1 \times 2$ structure. None of the other superstructures that are observed for an ion sputtered surface are forming on a fully oxidized sample. An STM image of this $1 \times 2$ structure is shown in figure 5 and appears to resemble the structure observed after high temperature annealing of the sputtered surface, shown in figure 3(g). Since both samples (i.e. the initially stoichiometric and the sputtered and oxygen depleted surface) eventually convert to a $1 \times 2$ surface structure, it may be concluded that this is the thermodynamically stable form of a reduced $\mathrm{SnO}_{2}(110)$ surface. The other structures, namely the $4 \times 1$ surface, appears to be a meta-stable $\mathrm{SnO}_{\mathrm{x}}$ overlayer with very low oxygen concentration that forms after oxygen depletion by ion sputtering. This overlayer is stable to high temperatures and thus appears to suppress the formation of the $1 \times 2$ structure. 

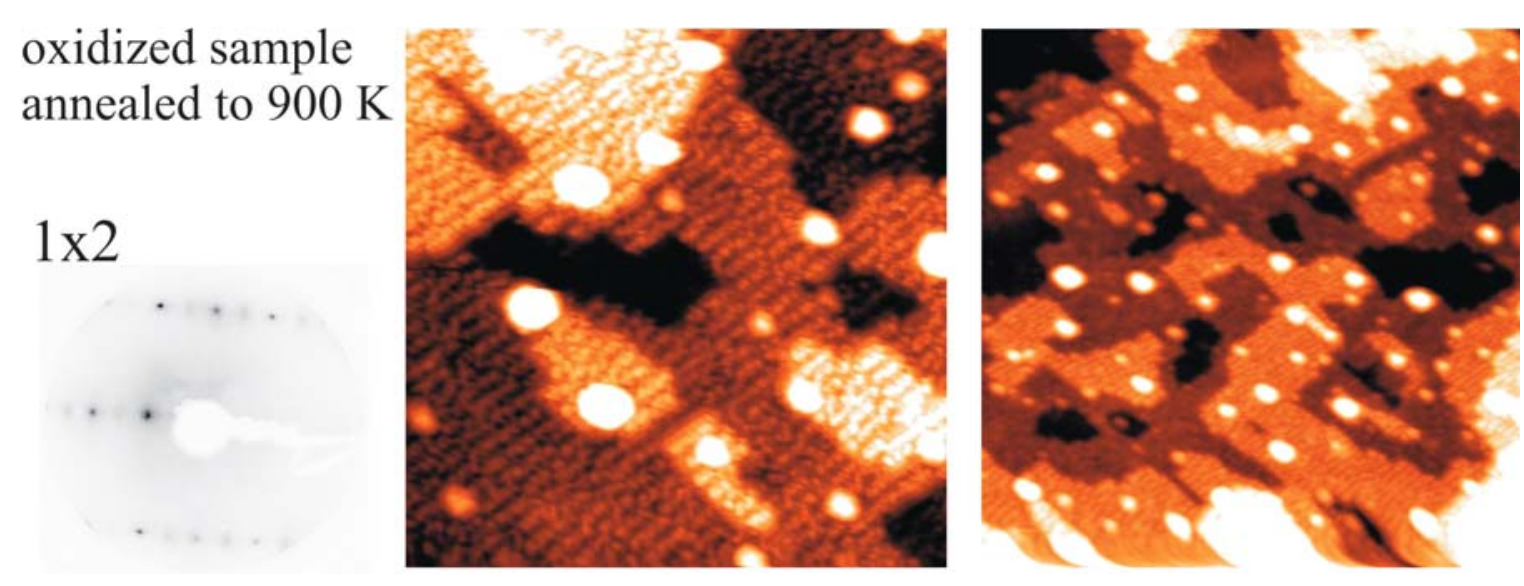

Figure 5. LEED and STM images of a 1x2 reconstructed surface obtained by vacuum annealing of a thoroughly oxidized $\mathrm{SnO}_{2}(110)$ sample to $\sim 950 \mathrm{~K}$ in vacuum.(reproduced with permission from ref.

The complexity of the (110) surface makes it a difficult model system for experimental surface science studies. The recent STM studies imply that even the "stoichiometric" $1 \times 1$ surfaces prepared at high pressure exhibit many poorly characterized defect structures. In addition the structures of the different surface reconstructions are controversial and in the opinion of this authors not resolved. In particular the $4 \times 1$ surface structure that can be easily prepared and shows a high degree of uniformity at the surface, would be a potentially intriguing model system. However, as long no reliable structural model exists any further surface science studies on this structure seem futile.

In the next subsection we describe the (101) surface which is better characterized and thus may provide a better model system.

\subsection{The (101) surface}

The (101) single crystal surface has been investigated just recently. [35,36,37,38,39] Apart from single crystal termination in an equilibrium crystal shape, it is also the dominant termination for $\mathrm{SnO}_{2}$ nanobelts [40] and is the face that grows epitaxially on r-cut alumina substrates.[41,42] The structural/compositional properties as well as its electronic properties have been investigated. These properties are being discussed in the following.

The dual valency of tin allows the formation of surfaces with a $\mathrm{Sn}^{2+}$ or a $\mathrm{Sn}^{4+}$ termination. The conversion from one Sn-charge state to the other is particularly easily accomplished on the (101) surface because of the atomic stacking in this crystallographic direction. Figure 6 shows a ball-andstick model of the $\mathrm{SnO}_{2}$ crystal structure. It can be seen that the atomic stacking along the (101) direction can be described as O-Sn-O trilayer. A stoichiometric sample will terminate in an oxygen layer and thus the surface Sn will retain its Sn(IV) bulk valency. However, it can be seen from figure 6 (b) that removal of the surface oxygen layer results in a Sn-O surface, where the surface Sn needs to adopt a Sn(II) charge state. Thus by simple addition or removal of the surface oxygen layer the surface Sn can convert from Sn(IV) to Sn(II) and vice versa. For the (110) surface the sample cannot easily convert from one Sn valency to another and this may be one reason for the complex surface reconstructions observed.29 On the (101) surface no reconstructions are necessary to go from a Sn(IV) 
to a Sn(II) surface. For either Sn-valency a (1x1) bulk truncation can be observed. Figure 7 shows LEED and STM of a Sn(II) surface prepared by ion sputtering and vacuum annealing. The atomically resolved STM image clearly indicates the 1x1 unit cell with Sn-atoms in the expected positions.

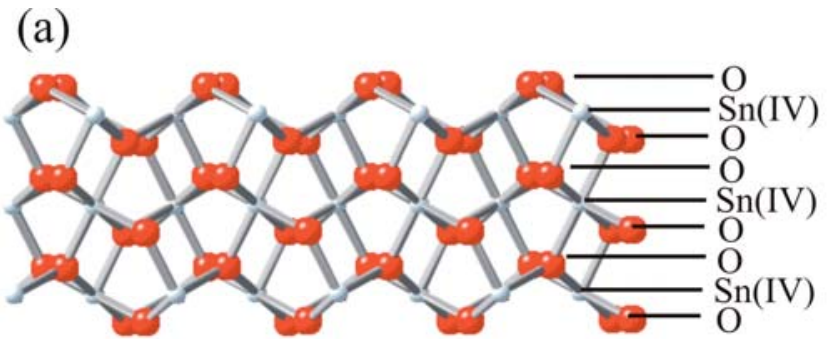

(b)

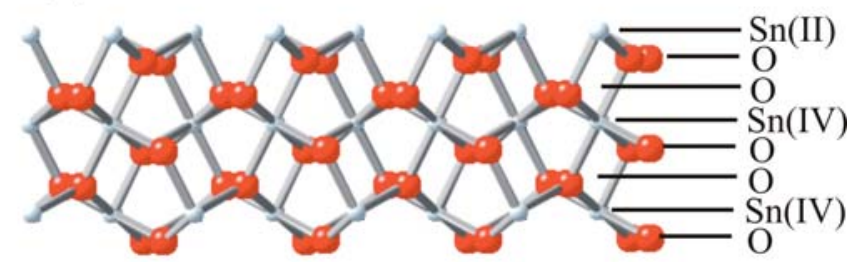

Figure 6. Ball-and-stick model of a cross-sectional view through a $\mathrm{SnO}_{2}$ crystal with a (101) orientation. The (101) surface is at the top. (a) Stoichiomtric bulk truncation with O termination. (b) reduced surface with Sn termination. In (b) surface Sn-atoms adopt a valency of II.

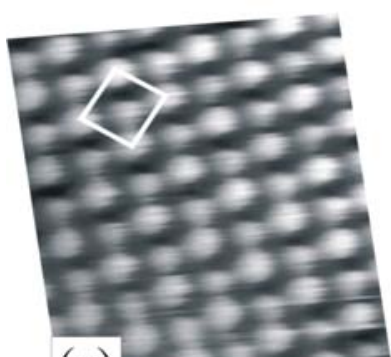

(a)
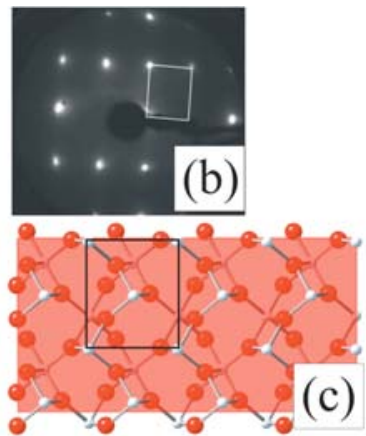

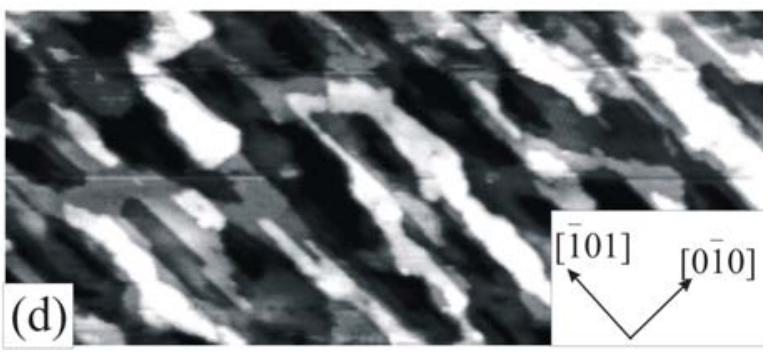

(d)

Figure 7. Surface structure of the $\mathrm{SnO}_{2}(101)$ surface. (a) Atomically resolved STM image with the unit cell indicated. (b) 1x1 LEED-pattern of $\mathrm{SnO}_{2}(101)$. Note the missing spots indicating the glideplane symmetry of the unit cell. (c) Ball-and-stick model of the surface with the unit cell indicated. (d) Large scale STM image of the surface showing the high density of step edges with preferential step edge orientation along the [-101] direction. (reproduced with permission from ref.[37]). 
Variable oxygen composition of oxide surfaces of multi-valent metals has been reported for other oxides.[43,44] The surface oxygen concentration in these oxides may depend on the oxygen chemical potential of the gas phase. In thermodynamic equilibrium the chemical potential of the surface is equilibrated to that of the surrounding atmosphere. Therefore, the oxygen concentration at the surface may be variable. The surface energy as a function of oxygen chemical potential of the system for different surface compositions of the $\mathrm{SnO}_{2}(101)$ surface was investigated by ab-initio thermodynamics calculations.[35,36] It was found that the reduced Sn(II) surface is indeed the lower energy surface for low oxygen chemical potential. Only above $\sim-1.7 \mathrm{eV}$ oxygen chemical potential the stoichiometric surface becomes more stable as can be seen in figure 8. In addition to the fully reduced and stoichiometric surfaces a small range of oxygen chemical potential was identified where a 1x2 surface structure is thermodynamically preferred. This superstructure consists of every other row of oxygen removed from the surface. Such a surface can be described by alternating double rows of Sn(II) and $\mathrm{Sn}(\mathrm{IV})$ and thus still satisfies the dual valency of $\mathrm{Sn}$.

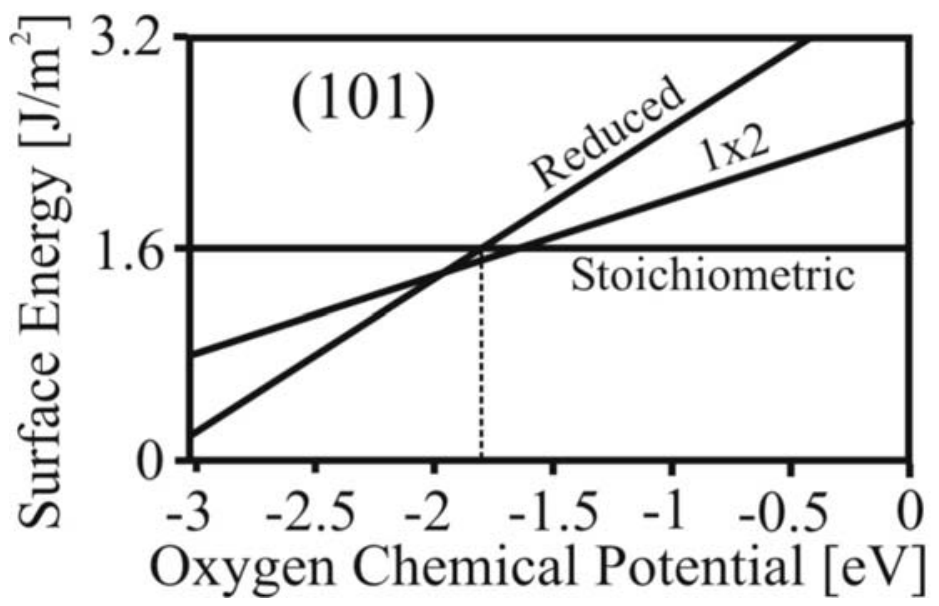

Figure 8. Ab-initio thermodynamic calculations showing the surface energy as a function of the oxygen chemical potential for the $\mathrm{SnO}_{2}$ (101) surface.(reproduced with permission from ref. [36]).

Experimentally the change in surface composition can be observed by vacuum annealing of a fully oxidized surface. This has been accomplished by exposing a vacuum cleaned surface to $10 \mathrm{mbar}_{2}$ at 500-600 K in a high pressure cell. Upon transfer of this sample into a UHV chamber the surface remains stoichiometric at low temperatures. Upon annealing the surface converts to a reduced surface at a temperature above $600 \mathrm{~K}$. The reduction of the surface was observed by low energy ion scattering (LEIS) $[35,36]$ and can also be observed by measuring the change in the surface electronic structure by photoemission spectroscopy. The latter method is shown in figure 9, on the example of angle resolved valence band spectroscopy at normal emission using synchrotron radiation with $34 \mathrm{eV}$ photon energy. At low temperatures the valence band exhibits bulk like features with a band gap (assuming the Fermiedge to be at the conduction band minimum) of close to the expected value of $3.6 \mathrm{eV}$ for bulk $\mathrm{SnO}_{2}$. With increasing temperature, surface reduction becomes apparent by the appearance of a band gap state with a maximum at a binding energy around $2.6 \mathrm{eV}$ referenced to the Fermi level. This state is becoming best defined at temperatures above $700 \mathrm{~K}$. In resonant photoemission studies it was demonstrated that this band gap state is Sn-derived.[36] Therefore it was concluded that reduction of 
the surface and consequently a transformation of surface Sn from Sn(IV) to Sn(II) results in the occupation of the mainly empty Sn-5s states in Sn(IV). These 5 s electrons form lone pair electrons at the surface and are associated with the band gap state on the reduced surface. In section 4.2 it is shown that these lone pair electrons are chemically inert towards the adsorption of water, this implies a generally low reactivity of the reduced surface towards similar molecules.

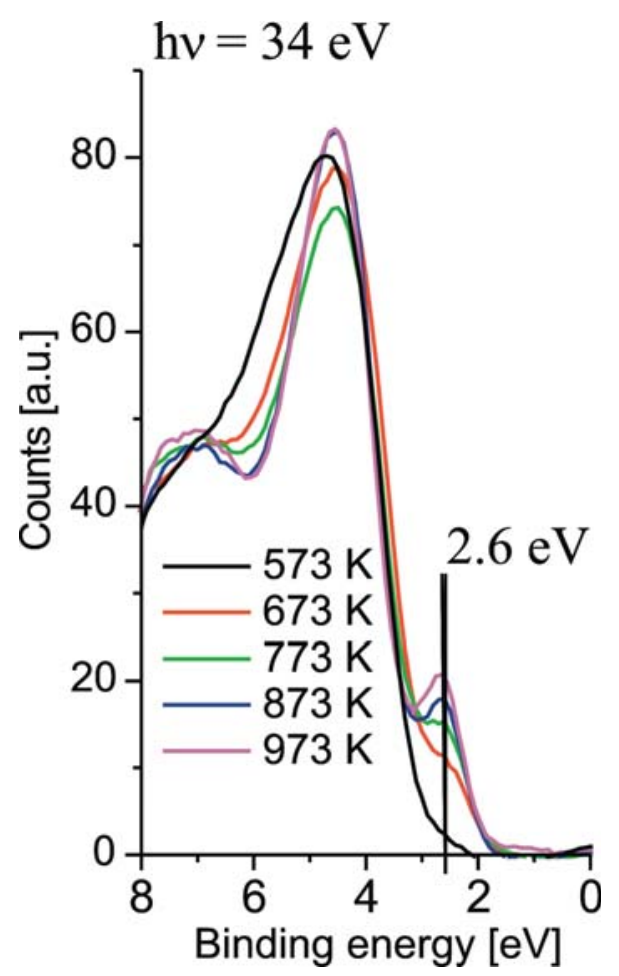

Figure 9. Evolution of the valence band spectrum of the $\mathrm{SnO}_{2}(101)$ surface with increasing annealing temperature. Above $600 \mathrm{~K}$ a band gap state develops at a binding energy of $2.6 \mathrm{eV}$. This band gap state is associated with Sn-5s electrons that become occupied as the surface reduces to form a Sn(II) surface layer.

In order to verify that the band gap states at the reduced surface are indeed only originating from the surface layer the dispersion of this state in angular resolved photoemission has been studied.[36] As expected, in normal emission the band gap state does not change in binding energy with varying photon-energy. Angle resolved photoemission spectra along three directions of the surface Brillouin zone are shown in figure 10. The dispersion of the band gap state and a second deeper lying state that has also been identified as a surface state are shown. The periodicity of the dispersion is in agreement with the dimensions of the surface Brillouin zone and thus the origin of this state as a surface feature is unambiguously established.

In context of gas sensors it is interesting to note that these Sn-5s states are low lying in the band gap and thus cannot contribute to the electrical conductivity of $\mathrm{SnO}_{2}$. Thus surface reduction by removal of lattice oxygen is unlikely to be the dominant mechanism for the increased conductivity of $\mathrm{SnO}_{2}$ gas sensors in a reducing atmosphere. However, the changed electronic and compositional features of the 
surface changes the interaction with adsorbates and this may be relevant for the gas response. The changed chemical properties will be demonstrated on the example of water adsorption in section 4.2.
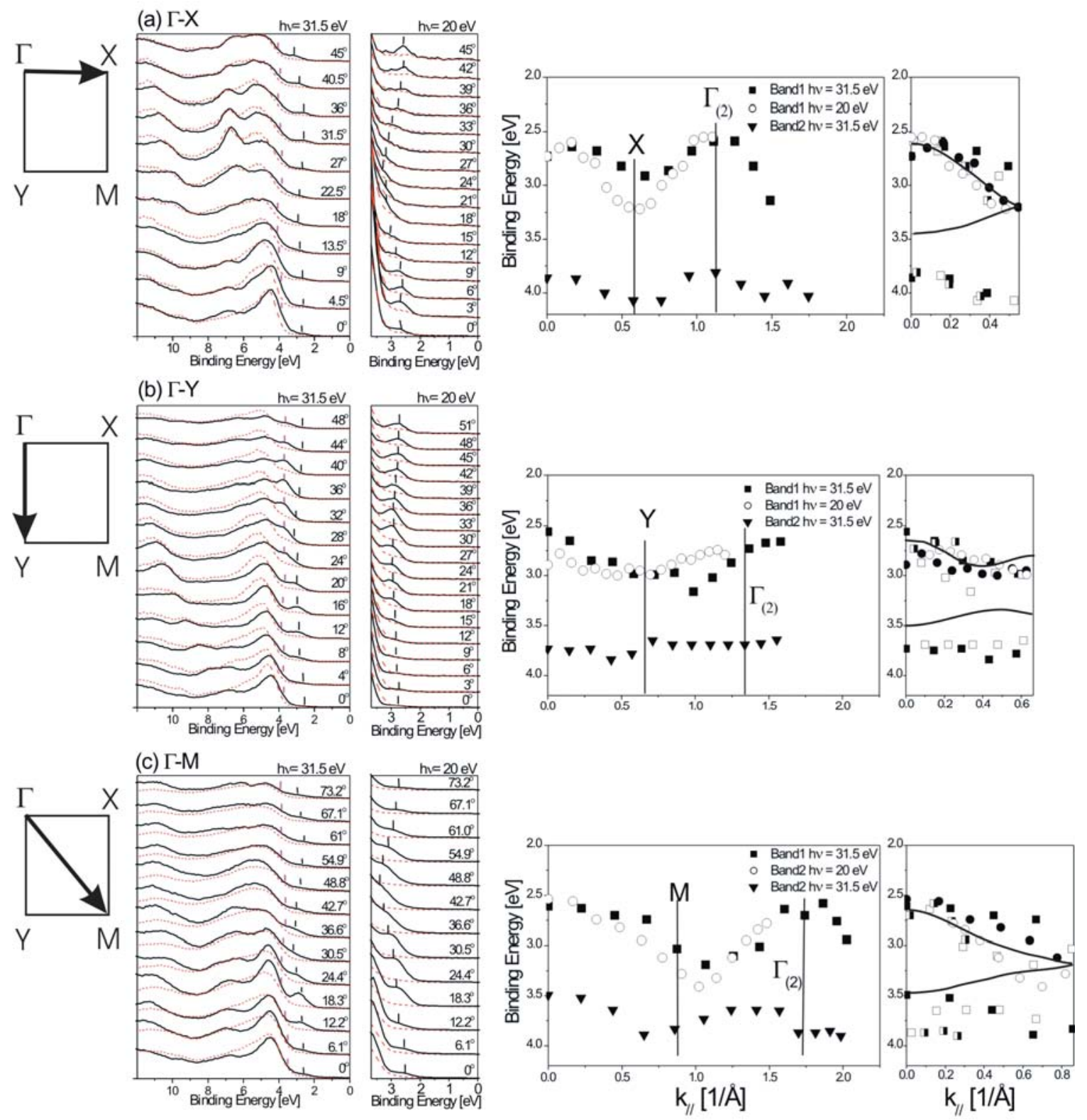

Figure 10. Angle-resolved photoemission measurements for the reduced (solid black lines) and stoichiometric (dashed red lines) $\mathrm{SnO}_{2}(101)$ surfaces along low symmetry directions of the surface Brillouin zone. On the right the dispersion of surface states for the reduced (101) surface are plotted. An effective electron mass of 0.7 times the free electron mass has been assumed to fit the symmetry of the surface Brillouin zone. A reduced zone-scheme is shown on the plots on the far right. The solid lines represent DFT-calculated surface states. (reproduced with permission from ref. [36]).

\section{Chemical properties of $\mathrm{SnO}_{2}$ surfaces on the example of water adsorption}

The omnipresence of water and the consequently unavoidable 'contamination' of any surface with water exposed to ambient conditions ensures continuous interest of surface scientists in the 
interaction of water with inorganic materials.[45,46] The adsorption of water on tin-dioxide surfaces is also of importance for its applications as a catalyst, electrode material, and as gas sensing material. For these applications the presence of water at the surface impacts device operations considerably. It has been reported for instance that water adsorption facilitates the catalytic conversion of $\mathrm{CO}$ to $\mathrm{CO}_{2}$ over $\mathrm{SnO}_{2}$ catalysts. Furthermore, many gas sensors are intended to detect harmful gases in the environment under ambient conditions and water is constantly present in these applications. In the use of $\mathrm{SnO}_{2}$ gas sensors it was early on recognized that $\mathrm{SnO}_{2}$ was not just sensitive to inflammable gases but also exhibites sensitivity to humidity.[47] This gas sensitivity sparked interest in the adsorption of water on $\mathrm{SnO}_{2}$ gas sensor materials. Water adsorption on pressed $\mathrm{SnO}_{2}$ powder or thin films prepared by spray pyrolysis was studied by transmission infrared spectroscopy,[48] temperature programmed desorption in a He-carrier gas,[49,50,51] and conductivity measurements.[50,52] Thornton and Harrison [53] found that molecular water was removed at $150{ }^{\circ} \mathrm{C}$ under atmospheric pressure, while hydroxyl groups started to desorb at $250{ }^{\circ} \mathrm{C}$ but some remained up to $500{ }^{\circ} \mathrm{C}$. These infrared spectroscopy results were taken to interpret later TPD studies, i.e. desorption peaks below $200{ }^{\circ} \mathrm{C}$ were assigned to desorption of molecular water and various high temperature desorption features were believed to originate from recombination of hydroxyls.[50,54] The TPD results differed for samples pre-annealed in a He-atmosphere from those annealed in oxygen.[54] More hydroxyl groups per unit surface area were found on the oxygen pretreated samples. In addition oxygen desorbed concurrent with water at high temperatures $\left(\sim 620^{\circ} \mathrm{C}\right)$ only for oxygen pretreated samples. This dependence on sample pretreatments suggested that oxygen vacancies and other defect sites at the surface may play an important role for water dissociation.[55] It is well established that water adsorption on $\mathrm{SnO}_{2}$ gas sensors increases its conductivity.[50,56,57,58,59,60] Yamazoe et al.[50] correlated the decrease in conductivity with the high temperature desorption peak in TPD and consequently concluded that hydroxyl groups cause the change in conductivity upon water adsorption on $\mathrm{SnO}_{2}$. The complex nature of the polycrystalline and powder samples considered makes it however impossible to make any definitive statement about water adsorption geometries and reactions. To obtain a better fundamental understanding of water adsorption on the stoichiometric $\mathrm{SnO}_{2}(110)$ surface as well as on the reduced and stoichiometric $\mathrm{SnO}_{2}(101)$ surfaces DFT calculations and experiments on single crystals have been recently performed. These studies are summarized in the following for two low index $\mathrm{SnO}_{2}$ surfaces.

\subsection{Water on the $\mathrm{SnO}_{2}(110)$ Surface}

First principle DFT calculations favor (partial) dissociative adsorption on the stoichiomteric, defect free (110) surface. Goniakowski and Gillan found that for $\mathrm{SnO}_{2}(110)$ dissociated water is the most stable configuration. [61] However, as pointed out by Lindan, [62] because inter-molecular bonding was not explicitly addressed in these calculations, these conclusions are of limited use. [63] 
Lindan studied half and full monolayer coverage of water and found that in both cases complete dissociation is the most energetically favored adsorption. However, while for half monolayer coverage no molecular adsorbed water was stable, at full coverage a half dissociated/ half molecular adsorbed water geometry was found to exhibit an only slightly lower adsorption energy compared to the fully dissociated structure. The considered adsorption geometries and the adsorption energies are shown in figure 11. Lindan also compared the results for $\mathrm{SnO}_{2}(110)$ with the much more frequently studied isostructural $\mathrm{TiO}_{2}(110)$ surface. He suggests that geometrical differences, i.e. a 5\% larger lattice constant of $\mathrm{SnO}_{2}$ compared to $\mathrm{TiO}_{2}$, is significant in explaining differences in water adsorption on these two surfaces. In his calculations for full monolayer water coverage at the $\mathrm{TiO}_{2}(110)$ surface, the mixed as well as the molecular adsorption states have a $0.1 \mathrm{eV}$ higher adsorption energy compared to the fully dissociated adsorption states. He explains this contrast between $\mathrm{TiO}_{2}$ and $\mathrm{SnO}_{2}$ by the larger separation between adsorbed water on $\mathrm{SnO}_{2}$ compared to water on $\mathrm{TiO}_{2}$. On $\mathrm{TiO}_{2}$ water can form $\mathrm{H}$-bonds between neighboring water molecules and thus stabilizes molecular adsorption, while on $\mathrm{SnO}_{2}$ such hydrogen bonding is less advantageous because of the greater separation. Bates [64] confirmed many of Lindan's conclusions for the full monolayer coverage on $\mathrm{SnO}_{2}(110)$ and extended the studies using a larger surface unit cell.

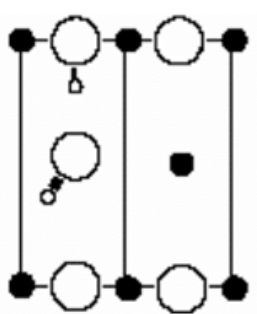

(a) $1.74 \mathrm{eV}$

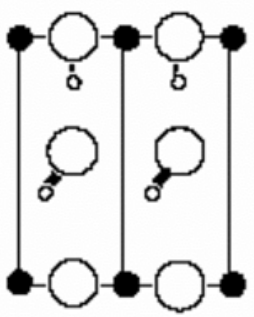

(d) $1.77 \mathrm{eV}$

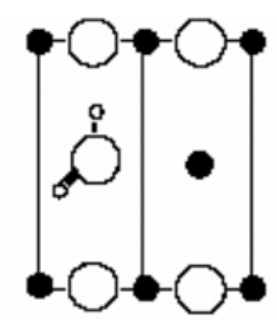

(b) Unstable

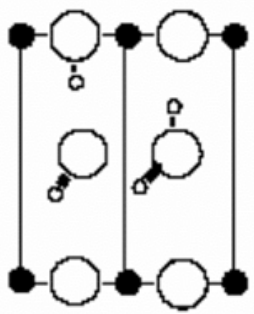

(e) $1.72 \mathrm{eV}$

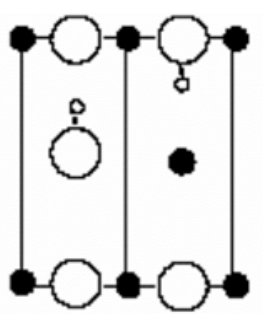

(c) $1.56 \mathrm{eV}$

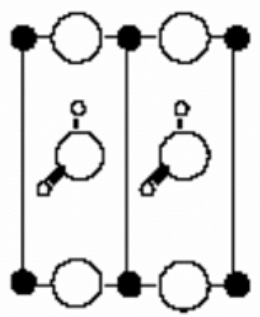

(f) Unstable

Figure 11. Schematic representation of the adsorption geometries of water on $\mathrm{SnO}_{2}(110)$ by DFT calculations. Oxygen, tin and hydrogen are represented by large, small filled and small open circles respectively. Half coverage (top) and monolayer coverage (bottom) are considered. Adsorption energies are also given. (reproduced with permission from ref. [62]).

Although all the theoretical studies point towards preferential dissociation of water on $\mathrm{SnO}_{2}(110)$ experimental studies have not unambiguously confirmed this conclusion. Gercher and Cox [65] performed UPS and TPD studies of water adsorption on $\mathrm{SnO}_{2}(110)$ single crystals. Molecular desorption peaks at 200 and $300 \mathrm{~K}$ were observed and desorption at $435 \mathrm{~K}$ was attributed to $\mathrm{OH}$ disproportionation. In their study they found only 10-15\% dissociated water on the stoichiometric, 
reduced (all bridging oxygen atoms removed) and highly-defective surfaces (with in plane oxygen vacancies). An increase in dissociation to about $35 \%$ was observed on a less defective surface. This suggested that a limited number of in-plane oxygen-vacancies promotes dissociation, but further increase in oxygen-vacancies decreases the dissociation probabilities. Although the samples were prepared with care it is very difficult to make quantitative statement about the defect concentration and structure on the (110) surface. [29] This also makes it questionable if the observed dissociated water is due to defects or occurs on perfect surfaces.

A downward band-bending of $\sim 0.1 \mathrm{eV}$ was observed for water adsorption on stoichiometric $\mathrm{SnO}_{2}(110)$ surfaces. [66] This implies an electron transfer from water to the substrate and is consistent with the increase in conductivity of $\mathrm{SnO}_{2}$ gas sensors in a humid atmosphere.

\subsection{Water on the $\mathrm{SnO}_{2}$ (101) Surface}

Water adsorption on the (101) surface was studied on both the stoichiometric and reduced bulk terminations (see figure 6) by UPS measurements and DFT calculations. [67] A strong difference in the water adsorption was observed depending on the oxidation state of the surface. Adsorption studies at $110 \mathrm{~K}$ are shown in Figure 12(a)-(c). The shift in the binding energy of the water molecular orbitals is a consequence of the different work function of the reduced versus stoichiometric surface. [68] On the stoichiometric surface weak additional features can be observed in the difference spectra (spectra that have the contribution of the clean surface subtracted from the water exposed samples) that may be assigned to orbitals of $\mathrm{OH}$ from dissociated water. A stronger difference is observed between the two surfaces if water is adsorbed at higher temperatures (160 K) (figure 12 (d) and (e)). At this temperature significant water adsorption is observed on the stoichiometric surface only. Furthermore, there is clear indication of dissociated water in the difference spectra for the stoichiomtric surface. In addition to valence band photoemission, band bending effects upon water adsorption was also investigated. Figure 12 (c) shows that water adsorption at $110 \mathrm{~K}$ causes a much stronger downward band bending on the stoichiomteric surface compared to the reduced surface. This is consistent with the notion that dissociated water causes most of the band bending and thus is responsible for the humidity gas response of $\mathrm{SnO}_{2}$.

The differences in the water adsorption on the reduced and stoichiometric $\mathrm{SnO}_{2}(101)$ surfaces has been verified by DFT calculations. Figure 12 (f)-(h) show the results of these calculations. On the reduced surface only molecular water is stable and adsorbs weakly, this is most likely because of the lack of surface oxygen that could accept hydrogen. For the stoichiometric surface both molecular and dissociative adsorption is possible, however, dissociation is thermodynamically favored and exhibits the highest adsorption energy of the configurations considered. 
(a)

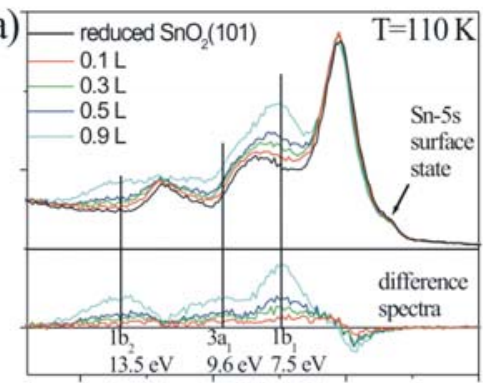

(b)

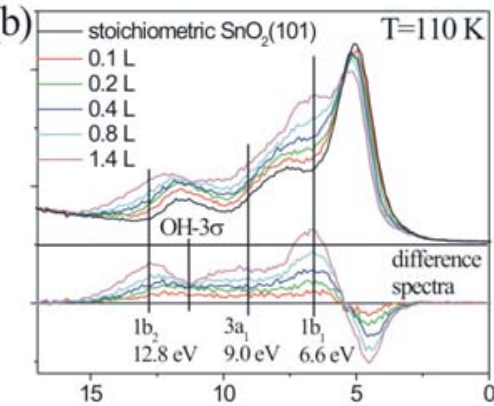

(c)

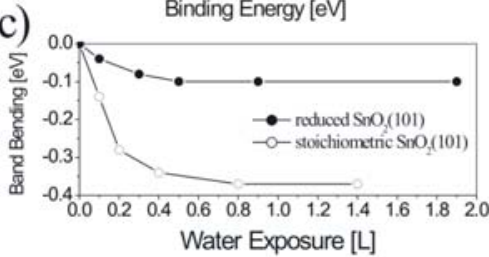

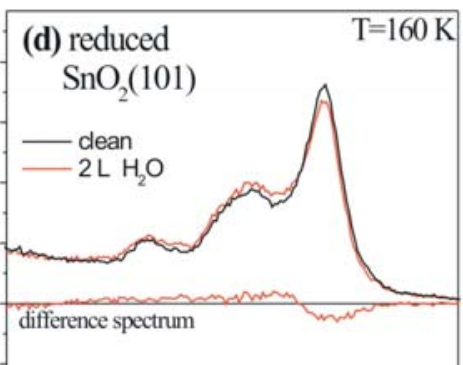

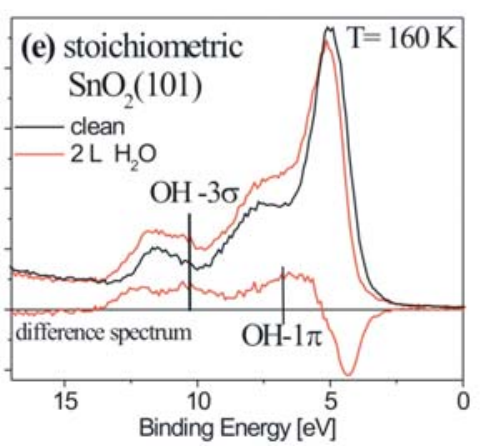

(f)

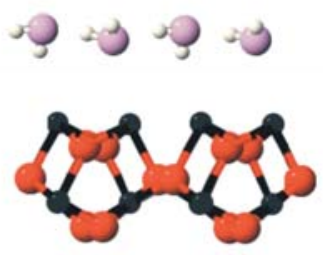

$-0.366 \mathrm{eV} /$ molecule

(g)

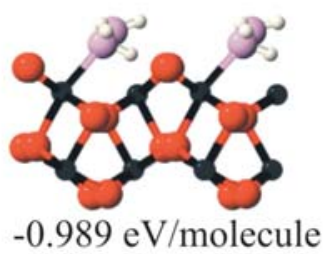

(h)

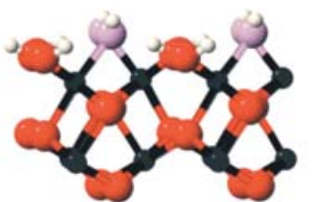

$-1.518 \mathrm{eV} / \mathrm{molecule}$

Figure 12. Water adsorption on the reduced and stoichiometric $\mathrm{SnO}_{2}(101)$ surface studied by UPS measurements and DFT calculations. Adsorption at $110 \mathrm{~K}$ is shown in (a)-(c) for the reduced surface (a) and stoichiometric surface (b). Band bending effects upon water exposure is plotted in (c) for the two surfaces. UPS measurements for water exposure at $160 \mathrm{~K}$ is shown in (d) for the reduced surface

and in (e) for the stoichiometric surface. Only on the stoichiometric surface (e) adsorption and dissociation of water is observed at $160 \mathrm{~K}$. Models of water adsorption derived from DFT calculations are shown in (f) for the reduced surface and (g) and (h) for the stoichiomteric surface for molecular and dissociative water adsorption, respectively. Calculated adsorption energies are also shown. (reproduced with permission from ref.[67]).

\section{Summary and final remarks}

We have shown that for $\mathrm{SnO}_{2}$ the surface composition is readily altered. The change in the surface oxygen composition is accompanied by a change in the electronic structure of surface Sn from a valency of (IV) for the stoichiometric surface to a valency of (II) for the reduced surface. This change in the composition also has a strong influence on its chemical and gas sensing properties. We showed for the adsorption of water that it dissociates on the stoichiometric surface but only adsorbs weakly on the reduced surface. While this easy reduction of $\mathrm{SnO}_{2}$ surface may play an important role in the gas sensing behavior of this material it is unlikely to be of general importance for other gas sensing metal oxides. The particular behavior of $\mathrm{SnO}_{2}$ surfaces arises from the dual valency of Sn. For other gas sensing materials like e.g. ZnO no variation in the lattice-oxygen composition is expected. 
In surface science one generally attempts to simplify the system in order to understand the fundamental processes. In this review we limited the description to single crystal $\mathrm{SnO}_{2}$ surfaces in order to understand structural, compositional, electronic, and chemical properties of tin oxide. Bulk single crystals are obviously no valid gas sensor materials, however, thin epitaxial films and single crystalline $\mathrm{SnO}_{2}$ nanobelts are. The surfaces of these materials should have the same properties as those discussed here for single crystalsl. Therefore the knowledge we gained is directly relevant for these gas sensing materials. Generally, gas sensors are more complex, however. Apart from their microstructure in porous films they are also often sensitized by a large variety of additives. Such additives are usually described as having two functions for the sensitivity of the gas sensor: (i) chemical sensitization and (ii) electronic sensitization. In the first case the additives essentially act as a catalyst that activates or dissociates the gas that is to be detected. For example hydrogen can be dissociated by a Pt-catalyst. Subsequent spill-over of atomic hydrogen to the tin oxide gas sensor causes then the gas response. In the second case the additive changes its chemical state, for example it is reduced by combustible gases. This change of the chemical state of the additive will change the interface electronic structure between the additive and the gas sensing material- causing "Schottkybarrier like” band bending effects and consequently a gas response. Again, although the basic principles seem to be understood there remain many open questions about the detailed functioning of these additives. For example, it is not known to what extend additives have to change their chemical state to induce a gas response and if there is any intermixing at the interface between additives and the gas sensing material. The complexity of most gas sensing materials makes it almost impossible to gain fundamental insight into the functioning of additives. Surface science investigations of clusters supported on single crystalline gas sensing materials is a possible way to gain the fundamental knowledge needed to suggestion strategies for improvements of gas sensors. Current surface science research is trying to understand systems that are already used; it will be a long way before new gas sensing materials are being designed on the basis of the understanding gained in surface science experiments.

\section{References and Notes}

1 Göpel, W. Chemisorption and charge transfer at ionic semiconductor surfaces: Implications in designing gas sensors. Prog. Surf. Sci. 1985, 20, 9-103.

2 Madou M.J.; Morrision S.R. Chemical Sensing with Solid State Devices Academic Press, Boston 1989.

3 Zhang D.; Liu Z.; Li C.; Tang T.; Liu X.; Han S.; Lei B.; Zhou C. Detection of $\mathrm{NO}_{2}$ down to ppb levels using undividual and multiple $\mathrm{In}_{2} \mathrm{O}_{3}$ nanowire devices. Nano Lett. 2004, 4, 1919-1924.

4 Mizokawa Y.; Nakamura S. ESR Study of Adsorbed Oxygen on Tin Dioxide. Oyo Buturi 1977, 46, 580-584.

5 Chang S.C. Oxygen chemisorption on tin oxide: Correlation between electrical conductivity and EPR measurements. J. Vac. Sci. Technol. 1980, 17, 366-369 .

6 Diebold U. The surface science of titanium dioxide. Surf. Sci. Rep. 2003, 48, 53-229.

7 Rantala T.T.; Rantala T.S.; Lantto V. Electronic structure of $\mathrm{SnO}_{2}(110)$ surface. Mater. Sci. Semicond. Process. 2000, 3, 103-107. 
8 Maki-Jaskari M.A.; Rantala T.T. Band structure and optical parameters of the $\mathrm{SnO}_{2}(110)$ surface. Phys. Rev. B 2001, 64, 075407-1 - 075407-7.

9 Maki-Jaskari M.A.; Rantala T.T. Theoretical study of oxygen-deficient $\mathrm{SnO}_{2}(110)$ surfaces. Phys. Rev. B 2002, 65, 245428-1 - 255428-8.

10 Rantala T.T.; Rantala T.S.; Lantto V. Surface relaxation of the (110) face of rutile $\mathrm{SnO}_{2}$. Surf. Sci. 1999, 420, 103-109.

11 Manassidis I.; Goniakowski J.; Kantorovich L.N; Gillan M.J. The structure of the stoichiometric and reduced $\mathrm{SnO}_{2}$ (110) surface. Surf. Sci. 1995, 339, 258-271.

12 Oviedo J.; Gillan M.J. Reconstructions of strongly reduced $\mathrm{SnO}_{2}(110)$ studied by first-principles methods. Surf. Sci. 2002, 513, 26-36.

13 Oviedo J.; Gillan M.J. The energetics and structure of oxygen vacancies on the $\mathrm{SnO}_{2}(110)$ surface Surf. Sci. 2000, 467, 35-48.

14 Sinner-Hettenbach M.; Gothelid M.; Weiß T.; Barsan N.; Weimar U.; von Schenck H.; Giovanelli L.; Le Lay G. Electronic structure of $\mathrm{SnO}_{2}\left(\begin{array}{lll}1 & 1 & 0\end{array}\right)-4 \times 1$ and sputtered $\mathrm{SnO}_{2}\left(\begin{array}{lll}1 & 1 & 0\end{array}\right)$ revealed by resonant photoemission. Surf. Sci. 2002, 499, 85-93.

15 Cox D.F.; Fryberger T.B.; Semancik S. Oxygen vacancies and defect electronic states on the $\mathrm{SnO}_{2}(110)-1$ x 1 surface. Phys. Rev. B. 1988, 38, 2072-2083.

16 Sinner-Hettenbach M.; Gothelid M.; Weissenrieder J.; von Schenck H.; Weiß T.; Barsan N.; Weimar U. Oxygen-deficient $\mathrm{SnO}_{2}(110)$ : a STM, LEED and XPS study. Surf. Sci. 2001, 477, 50 58.

17 Themlin J.M; Sporken R.; Darville J.; Caudano R.; Gilles J.M. Resonant-photoemission study of $\mathrm{SnO}_{2}$ : Cationic origin of the defect band-gap states. Phys. Rev. B 1990, 42, 11914-11925.

18 Egdell R.G.; Erickson S.; Flavell W.R. Oxygen deficient $\mathrm{SnO}_{2}(110)$ and $\mathrm{TiO}_{2}(110)-\mathrm{a}$ comperative-study by photoemission. Solid State Commun. 1986, 60, 835-838.

19 Cox D.F; Semancik S.; Szuromi P.D. Structural and electronic-properties of clean and water dosed $\mathrm{SnO}_{2}$ (110). J. Vac. Sci. Technol. A 1986, 4, 627-628.

20 Cox D.F.; Fryberger T.B.; Erickson J.W.; Semancik S. Surface-properties of clean and gas-dosed $\mathrm{SnO}_{2}$ (110). J. Vac. Sci. Technol. A 1987, 5, 1170-1171.

21 de Fresart E.; Darville J.; Gilles J.M. Influence of the surface reconstruction on the work function and surface conductance of (110)SnO $\mathrm{Sn}_{2}$. Appl. Surf. Sci. 1982, 11/12, 637-639.

22 Cox D.F.; Fryberger T.B. Preferential isotopic labeling of lattice oxygen positions on the $\mathrm{SnO}_{2}(110)$ surface. Surf. Sci. 1990, 227, L105- L108.

23 Atrei A.; Zanazzi E.; Bardi U.; Rovida G. The $\mathrm{SnO}_{2}(110)(4 \times 1)$ structure determined by LEED intensity analysis. Surf. Sci. 2001, 475, L223- L228.

24 Jones F.H.; Dixon R.; Foord J.S; Egdell R.G.; Pethica J.B. The surface structure of $\mathrm{SnO}_{2}(110)$ (4x1) revealed by scanning tunneling microscopy. Surf. Sci. 1997, 376, 367-373.

25 Pang C.L.; Haycock S.A.; Raza H.; Møller P.J.; Thornton G. Structures of the 4x1 and 1x2 reconstructions of $\mathrm{SnO}_{2}(110)$. Phys. Rev. B 2000, 62, R7775- R7778.

26 Abee M.W.; Cox D.F. $\mathrm{NH}_{3}$ chemisorption on stoichiometric and oxygen-deficient $\mathrm{SnO}_{2}(110)$ surfaces. Surf. Sci. 2002, 520, 65- 77. 
27 Abee M.W.; Cox D.F. $\mathrm{BF}_{3}$ adsorption on stoichiometric and oxygen-deficient $\mathrm{SnO}_{2}(110)$ surfaces. J. Phys. Chem. B 2003, 107, 1814-1820.

28 Gercher V.A.; Cox D.F. Formic acid decomposition on $\mathrm{SnO}_{2}(110)$. Surf. Sci. 1994, 312, 106- 114.

29 Batzill M.; Katsiev K.; Diebold U. Surface morphologies of $\mathrm{SnO}_{2}(110)$. Surf. Sci. 2003, 529, 295311.

30 Themlin J.-M.; Gilles J.-M.; Johnson R.L. Oxygen 2s spectroscopy of tin oxides with synchrotron radiation-induced photoemission. J. Physique IV 1994, 4, C9- C12.

31 Cox D.F.; Fryberger T.B.; Semancik S. Oxygen vacancies and defect electronic states on the $\mathrm{SnO}_{2}(110)-1$ x 1 surface. Phys. Rev. B 1988, 38, 2072-2083.

32 Gercher V.A.; Cox D.F.; J.-M. Themlin, Oxygen-vacancy-controlled chemistry on a metal-oxide surface-methanol dissociation and oxidation on $\mathrm{SnO}_{2}(110)$. Surf. Sci. 1994, 306, 279-293.

33 Gercher V.A.; Cox D.F. Water-adsorption on stoichiometric and oxygen deficient $\mathrm{SnO}_{2}(110)$ surfaces. Surf. Sci. 1995, 322, 177-184.

34 Cavicchi R.; Sukkarev V.; Semancik S. Preparation of well-oredered, oxygen-rich $\mathrm{SnO}_{2}(110)$ surfaces via oxygen plasma treatment. J. Vac. Sci. Technol. A 1990, 8, 2347-2352.

35 Batzill M.; Chaka A. M.; Diebold U. Oxygen chemistry of a gas sensing material: $\mathrm{SnO}_{2}$ (101). Europhys. Lett. 2004, 65, 61-65.

36 Batzill M.; Katsiev K.; Burst J.M.; Diebold U.; Chaka A.M.; Delley B. Gas phase-dependent properties of $\mathrm{SnO}_{2}$ (110), (100), and (101) single crystal surfaces: structure, composition, and electronic properties. Phys. Rev. B 2005, 72, 165414-1 - 165414-20.

37 Batzill M.; Diebold U. The surface and materials science of tin oxide. Prog. Surf. Sci 2005, 79, 47-154.

38 Batzill M.; Diebold U. Characterizing solid state gas responses by surface charging in photoemission: Water adsorption on $\mathrm{SnO}_{2}(101)$. J. Phys.: Condens. Matter 2006, 18, L1-L4.

39 Batzill M.; Katsiev K.; Burst J.M.; Losovyj Y.; BergermayerW.; Tanaka I.; Diebold U. Tuning surface properties of $\mathrm{SnO}_{2}(101)$ by reduction. J. Phys. Chem. Mater. 2006, 67, 1923-1929.

40 Dai Z.R.; Pan Z.W.; Wang Z.L. Ultra-long single crystalline nanoribbons of tin oxide. Solid State Commun. 2001, 118, 351-354.

41 Cavicchi R. E; Semancik S.; Antonik M. D.; Lad R.J. Layer-by-layer growth of epitaxial $\mathrm{SnO}_{2}$ on sapphire by reactive sputter deposition. Appl. Phys. Lett. 1992, 61, 1921-1923.

42 Batzill M.; Burst J. M.; Diebold U. Pure and Co-doped $\mathrm{SnO}_{2}(101)$ films grown by $\mathrm{MBE}$ on $\mathrm{Al}_{2} \mathrm{O}_{3}$. Thin Solid Films 2005, 484, 132-139.

43 Weiss W.; Ranke W. Surface chemistry and catalysis on well-defined epitaxial iron-oxide layers. Prog. Surf. Sci. 2002, 70, 1-151.

44 Surnev S.; Ramsey M.G.; Netzer F.P. Vanadium oxide surface studies. Prog. Surf. Sci. 2003, 73, 117-165.

45 Henderson M.A. The interaction of water with solid surfaces: fundamental aspects revisited. Surf. Sci. Rep. 2002, 46, 1-308.

46 Thiel P.A.; Madey T.E. The interaction of water with solid surfaces: Fundamental aspects. Surf. Sci. Rep. 1987, 7, 211-385. 
47 Boyle J.F.; Jones K.A The Effects of CO, water vapor and surface temperature on the conductivity of a $\mathrm{SnO}_{2}$ gas sensor. J. Electronic Mater. 1977, 6, 717-720.

48 Tamaki J.; Nagaishi M.; Teraoka Y.; Miura N.; Yamazoe N.; Moriya K.; Nakamura Y. Adsorption behavior of $\mathrm{CO}$ and interfering gases on $\mathrm{SnO}_{2}$. Surf. Sci. 1989, 221, 183-186.

49 Thornton E.W.; Harrison P.G. Tin oxide surfaces. J. Chem Soc. Faraday Trans. 1 1975, 71, 461465.

50 Yamazoe N.; Fuchigami J.; Kishikawa M.; Seiyama T. Interactions of tin oxide with $\mathrm{O}_{2}, \mathrm{H}_{2} \mathrm{O}$ and $\mathrm{H}_{2}$. Surf. Sci. 1979, 86, 335-344.

51 Tamaki J.; Nagaishi N.; Teraoka Y.; Miura N.; Yamazoe N.; Moriya K.; Nakamura Y. Adsorption behavior of $\mathrm{CO}$ and interfering gases on $\mathrm{SnO}_{2}$. Surf. Sci. 1989, 221, 183-196.

52 Korotchenkov G.; Brynzari V.; Dmitriev S. Electrical behavior of $\mathrm{SnO}_{2}$ thin films in humid atmosphere. Sens. Actuators B 1999, 54, 197-201.

53 Thornton E.W.; Harrison P.G. Tin oxide surfaces. Part 3.--Infrared study of the adsorption of some small organic molecules on tin(IV) oxide. J. Chem. Soc. Faraday Trans. I 1975, 71, 24682472.

54 Egashira M.; Nakashima M.; Kawasumi S.; Seiyama T. Temperature programmed desorption study of water adsorbed on metal oxides: 2. tin oxide surfaces. J. Phys. Chem. 1981, 85, 41254130.

55 Egashira M.; Kawasumi S.; Kagawa S.; Seiyama T. Temperature programmed desorption study of water adsorbed on metal oxides: I anatase and rutile. Bull. Chem. Soc. Jpn. 1978, 51, 3144-3146.

56 Williams D.E. In Solid State Gas Sensors; Moseley P.T.; Tofield B.C., Eds.; Adam Hilger IOP, Publishing 71 (1987) Chapter 5.1.

57 Barsan N.; Ionescu R. The mechanism of the interaction between $\mathrm{CO}$ and an $\mathrm{SnO}_{2}$ surface: the role of water vapour. Sensors and Actuators B 1983, 12, 71-75.

58 Ionescu R.; Vancu A.; Moise C.; Tomescu A. Role of water vapour in the interaction of $\mathrm{SnO}_{2}$ gas sensors with $\mathrm{CO}$ and $\mathrm{CH}_{4}$. Sensors and Actuators B 1999, 61, 39-42.

59 Hahn S.H.; Bârsan N.; Weimar U.; Ejakov S.G.; Visser J.H.; Soltis R.E. CO sensing with $\mathrm{SnO}_{2}$ thick film sensors: role of oxygen and water vapour. Thin Solid Films 2003, 436, 17-24.

60 Schierbaum K.D.; Wiemhöfer H.D.; Göpel W. Defect structures and sensing mechansism of $\mathrm{SnO}_{2}$ gas sensors: comparative electrical and spectroscopic studies. Solid State Ion. 1988, 28-30, 16311636.

61 Goniakowski J.; Gillan M.J. The adsorption of $\mathrm{H}_{2} \mathrm{O}$ on $\mathrm{TiO}_{2}$ and $\mathrm{SnO}_{2}(110)$ studied by firstprinciples calculations. Surf. Sci. 1996, 350, 145-158.

62 Lindan P.J.D. Water chemistry at the $\mathrm{SnO}_{2}(110)$ surface: the role of inter-molecular interactions and surface geometry. Chem. Phys. Lett. 2000, 328, 325-329.

63 Lindan P.J.D.; Harrison N.M.; Gillan M.J. Mixed Dissociative and Molecular Adsorption of Water on the Rutile (110) Surface. Phys. Rev. Lett. 1998, 80, 762-765.

64 Bates S.P. Full-coverage adsorption of water on $\mathrm{SnO}_{2}(110)$ : the stabilization of the molecular species. Surf. Sci. 2002, 512, 29-36.

65 Gercher V.A.; Cox D.F. Water adsorption on stoichiometric and defective $\mathrm{SnO}_{2}(110)$ surfaces. Surf. Sci. 1995, 322, 177-184. 
66 Semancik S.; Cox D.F. Fundamental characterization of clean and gas-dosed tin oxide. Sensors and Actuators 1987, 12, 101-106.

67 Batzill M.; Bergermayer W.; Tanaka I.; Diebold U. Tuning the chemical response of a gas sensitive material: Water adsorption on $\mathrm{SnO}_{2}$ (101). Surf. Sci. 2006, 600, L 29-L32.

68 Batzill M.; Katsiev K.; Diebold U. Tuning the oxide/organic interface: Benzene on $\mathrm{SnO}_{2}(101)$. Appl. Phys. Lett. 2004, 85, 5766-5768.

(C) 2006 by MDPI (http://www.mdpi.org). Reproduction is permitted for noncommercial purposes. 